\title{
THE VALUE OF DYNAMIC CONTRAST ENHANCED BREAST MRI IN MAMMOGRAPHICALLY DETECTED BI-RADS 5 MICROCALCIFICATIONS
}

\author{
Dana Houserkova ${ }^{a *}$, Sachin Prasad N ${ }^{\mathrm{a}}$, Ivan Svach ${ }^{\mathrm{c}}$, Ladislava Kucerova ${ }^{\mathrm{b}}$, Milada Duskova ${ }^{\mathrm{b}}$, \\ Jiri Bucil $^{\mathrm{a}}$, Ivan Sisola ${ }^{\mathrm{a}}$, Nora Zlamalova ${ }^{\mathrm{c}}$, Hana Svebisova ${ }^{\mathrm{d}}$
}

a Department of Radiology, Faculty of Medicine and Dentistry, Palacky University, 77515 Olomouc, Czech Republic

${ }^{b}$ Department of Pathology, Faculty of Medicine and Dentistry, Palacky University, 77515 Olomouc

c Department of Surgery, Faculty of Medicine and Dentistry, Palacky University, 77515 Olomouc

${ }^{d}$ Department of Oncology, Faculty of Medicine and Dentistry, Palacky University, 77515 Olomouc

e-mail:dana.houserkova@seznam.cz

Received: November 11, 2007; Accepted (with revisions): February 8, 2008

Key words: Microcalcification/MRI - mammography/Ductal carcinoma in situ/Multifocality

Aims: To evaluate the role of dynamic contrast-enhanced magnetic resonance imaging (MRI) in the diagnosis of BI-RADS-5 type of microcalcifications of the breast, to compare the size of the microcalcification lesions using mammography (MG) and MRI, and to determine the value of MRI in surgery for microcalcifications. The study also determines the morphology of microcalcification lesions, assesses kinetic curves and compare MRI features of ductal carcinoma in situ (DCIS) for different histopathological grades.

Methods: Our group consisted of 32 patients with mammographically detected BI-RADS 5 microcalcifications. The MRI was done in this group of women which was later followed by stereotactic vaccum-assisted biopsy (SVAB). Surgery was performed on all patients with a biopsy that resulted in a diagnosis of breast cancer or atypical ductal hyperplasia (ADH).

Results: Of our group of 32 patients, there were 35 mammograhically detected microcalcification lesions, 32 DCIS, one ADH and two benign findings according to the final histology.

The microcalcification lesions were larger using MRI than in MG in 10 women. We diagnosed DCIS multifocality in 6 women and bilateral carcinoma in one woman. As with kinetic curve assessment, we found in $67 \%$ of DCIS a rapid rise, $27 \%$ a moderate and in $6 \%$ a slow initial rise. With the pattern of enhancement in the delayed phase, we found in $30 \%$ of DCIS a washout pattern, $67 \%$ a plateau and in $3 \%$ a persistent pattern. Noted difference between high and low grade DCIS was confirmed.

Conclusions: MRI sensitivity in the detection of DCIS was $94 \%$ in our group of patients and was the sole evidence for detection of multifocality and bilateral incidence of carcinoma. In $26 \%$ of women the outcome of MRI was the most important for converting breast conserving surgery to mastectomy.

\section{INTRODUCTION}

Magnetic resonance imaging (MRI) is a method also used in breast imaging. It has changed its role recently with the development of modern scanners and new sequences. Breast MRI is an imaging method with big importance not only concerning the morphology of the lesion, but also the evaluation of its functional parameters. The evaluation of the enhancement from the quantitative and qualitative points of view, is in fact the assessment of vascularization of the lesion. The attribute of neoangiogenesis is used in malignant lesions which are often too small to be proved by another imaging method.

The value of breast MRI for ductal carcinoma in situ (DCIS) detection is being discussed. The most common presentations of DCIS are the malignant microcalcifications in mammography (MG). In the case of DCIS manifesting by microcalcification in mammography, the real advantage of breast MRI is the ability to evaluate accurately the microcalcification lesion and even the mam- mographically occult area without microcalcifications by detecting the DCIS with microinvasion. The great benefit of preoperative breast MRI is in the evaluation of multifocality and bilateral incidence of carcinoma.

The aim of our study was the following:

1) to evaluace the sensitivity, accuracy, positive predictive value (PPV) and negative predictive value (NPV) of dynamic contrast-enhanced breast MRI for evaluation of malignancy in BIRADS-5 (Breast Imaging Reporting and Data System) microcalcifications.

2) to compare the size of the microcalcification lesions in MG and MRI.

3) to determine the value of MRI for evaluating the multifocality and bilateral incidence of carcinoma.

4) to determine the MRI morphologic features and to find out the enhancement parameters in microcalcification lesions.

5) to compare MRI enhancement parameters in DCIS of different histopathological grades. 
6) to assess the benefit of breast MRI for following surgical therapy.

\section{MATERIALS AND METHODS}

\section{Patient population}

Consecutive patients with mammographically detected BI-RADS 5 microcalcifications and mammographically dense type of breast (type 4 and 5 according to Tabár ${ }^{1,2}$ ) were recruited from January 2004 to December 2006. Three women with contraindications to MRI (with claustrophobia or pacemaker) were excluded. These entrance criterias fulfilled 32 women, aged $34-72$ years ( 50.5 years of age in an average). In all women, after mammography we performed an ultrasound examination and contrast dynamic-enhanced MRI of the breast prior to stereotactic vaccum-assisted biopsy (SVAB).

\section{Mammography protocol}

Bilateral mammography was performed (Diamond, Instrumentarium Imaging, Tuusula, Finland) and included craniocaudal and mediolateral oblique view of the breasts and spot - magnification views over the area of microcalcifications. The mammograms were independently double-read using BI-RADS assesment categories by two or four radiologists with 5-15 years of experience in mammography. Radiologists also scored breast density according to Tabár's classification (1-5). Microcalcifications were classified according to BI-RADS for mammographic features including calcification morphology and distribution.

\section{Breast ultrasound protocol}

In all women, bilateral breast ultrasound (US) was perfomed with the knowledge of mammographic findings of BI-RADS 5 microcalcificatons. US using a linear-array transducer with a center frequency of $8-11 \mathrm{MHz}$ was performed by the same group of radiologists as mammography in breast US (Logiq $500 \mathrm{MD}$, General Electric, Solingen, Germany). We were able to detect the area of microcalcifications in breast in 12 women, but we found no mass. In the remaining 23 women, the microcalcificatios were not seen in ultrasound.

\section{Breast MRI protocol}

In premenopausal women, we performed breast MRI within the second week of the menstrual cycle to avoid contrast uptake due to hormonal dependent changes of the breast. We examined these women in prone position with their breasts fixed in a double breast coil. The scanner was a $1.5 \mathrm{~T}$ system (Siemens Symphony, Erlangen, Germany, Siemens). Using double breast coil enabled comparison of both breast in one image. Image protocol included a localizer followed by transverse turbo-spin echo T2-weighted sequence (TR/TE, 4430/97 ms, matrix $512 \times$ 512) and by coronal STIR (Short Tau Inversion Recovery) sequence (TR/TE, 9370/70 ms). Other parameters were section thickness $3 \mathrm{~mm}$ and interslice gap $0.8 \mathrm{~mm}$. These sequences were followed by a dynamic study consisting of serial imaging of three-dimensional transverse fast low angle shot T1-weighted sequence (TR/TE, 4.42/1.67; matrix, $512 \times 512$; section thickness $1.2 \mathrm{~mm}$ ) with fat supression. For dynamic study we acquired one pre- and six post-contrast sequences. Contrast media Prohance (Gadoteridol, $0.5 \mathrm{M}$ ) was administered immediately after the end of first (precontrast) sequence as a bolus intravenous injection at a dose of $0.1 \mathrm{mmol} / \mathrm{kg}$ body weight and was followed by a $20 \mathrm{ml}$ saline solution. The total time of the dynamic study was 6.26 minutes. The necessary part of the examination was postprocessing by creating subtraction images of each postcontrast sequence and by creating maximum intensity projection (MIP) images.

For dynamic contrast-enhanced breast MRI, a malignant lesion was considered to be diagnosed successfully (true positive) if it showed contrast uptake in the area of microcalcification. The absence of contrast uptake in the area of microcalcification that was assessed to be malignant in SVAB or surgery was classified as false-negative MRI findings. Sensitivity, specificity, accuracy, positive predictive value and negative predictive value were calculated on the basis of the absence or presence of contrast uptake in the area of BI-RADS 5 microcalcification.

MRI morphology of enhancement and kinetic curves were evaluated for all the 31 enhanced microcalcification lesions. We evaluated two main morphological MRI patterns of enhanced microcalcification lesions. By the quantitative evaluation, we assessed the increase in signal intensity on the second post-contrast sequence compared to the pre-contrast sequence. We determined three levels of intensity of enhancement, slow (1st level, 0-50\%), medium (2nd level, 50-100\%) and the rapid (3rd level, above $100 \%$ ). For qualitative evaluation we used time to intensity curves (TIC) for this evaluation. We differentiated three types of enhancement patterns (three types of TIC). Curve type 1 - "persistent pattern", with a continuous increase in signal intensity on each successive contrast-enhanced image. Type 2 - "plateau pattern", in which an initial increase in signal intensity was followed by a flattening and fluctuation of the enhancement curve $( \pm 10 \%)$. Type 3 - "washout pattern", with an initial increase and subsequent decrease in signal intensity. We evaluated the initial rise of enhancement and type of enhancement pattern with regard to the final histology in microcalcification lesions. We used chi-square test to compare the differences in enhancement parameters in DCIS of various histopathological grades.

Each examination was evaluated by radiologist with 4 years experience in breast MRI.

\section{SVAB protocol}

SVAB using 11 or 8-gauge probes (Mammotome, Ethicon Endo-Surgery, Cincinnati, USA) was performed. A radiologist with 5 years experience in SVAB performed the biopsy. The average number of samples taken during the biopsy was 15 . Specimen radiography was performed routinely on all samples. Complete or partial removal of the microcalcifications was assessed in all cases on two 
view mammograms immediately. If the microcalcifications were removed completely, clips were placed through the probe to identify the SVAB site for subsequent surgical excision. The histopathological result was correlated with the mammographic findings by radiologist.

\section{Histological diagnosis}

Histological diagnosis was determined by two pathologists with 10-20 years of experience in breast histology. The histological findings were classified into three groups - malignant, high risk and benign. Malignant lesions included invasive carcinoma and DCIS. The grade of DCIS was scored as low, intermediate or high. Atypical ductal hyperplasia $(\mathrm{ADH})$ was considered to be high-risk lesion for which, the associated presence of carcinoma can be underestimated with SVAB. Lesions that were not classified as histologically malignant or high risk were categorized as benign. All women with malignant or high risk microcalcification lesions in SVAB were operated. If malignancy was found in SVAB, the surgeon performed a therapeutic operation including axillary surgery if indicated. In case of ADH the surgeon performed surgical excision. If a benign microcalcification lesion was found in SVAB, the woman was followed by mammography of the ipsilateral breast at 6 months interval. If there was no calcification progression in post-SVAB mammographic follow-up, mammography was recommended in usual screening interval.

\section{RESULTS}

The histopathological report of our group of 32 patients (aged between 34-72 years) revealed 31 lesions with DCIS diagnosed in SVAB, two with ADH and two with benign findings. 33 lesions with malignant or suspicious finding from previous biopsy were operated. SVAB, histologic findings and the subsequent surgical excision were compared.

Final histology after surgery confirmed 32 DCIS lesions. Among them, 22 pure DCIS were found and in 10 DCIS with microinvasion was proved. One ADH was upgraded to DCIS after surgery and in one ADH was cofirmed by open biopsy. Regarding the histopathological grade of pure DCIS, high-grade DCIS was confirmed in 7 women, intermediate-grade DCIS in 9 women and lowgrade DCIS in 6 women. Two women with benign microcalcification lesions in SVAB were followed at 6 months interval and no false-negative diagnosis occured, there was no microcalcification progression at mammographic follow-up.

In two benign microcalcification lesions, there was no contrast enhancement in breast MRI. In one case of $\mathrm{ADH}$ we found contrast uptake in the microcalcification lesion. Dynamic contrast-enhanced MRI showed uptake of contrast in the area of microcalcifications in 31 (94\%) with DCIS. In 2 (6 \%) women with histologically proved low grade DCIS, we could not assess the contrast enhancement in breast MRI and the result of breast MRI did not prove the existence of DCIS. The sensitivity of contrast-enhanced breast MRI in assessment of BI-RADS 5 microcalcification lesion was $94 \%$, the accuracy $94 \%$, PPV $100 \%$ and NPV $50 \%$.

Regarding the size of microcalcification lesion in breast MRI, we proved the same size of lesion in comparison to MG in 21 (68\%) patients, and in 10 (32\%) patients the size of enhancement in MR imaging was larger - up to $10 \mathrm{~mm}$ in 2 patients, up to $15 \mathrm{~mm}$ in 3 patients and up to $20 \mathrm{~mm}$ in 5 women.

Multifocality of DCIS was found by MRI in 6 (19\%) women (Fig. 1). Bilateral carcinoma was found in one of these patients.

In 31 contrast-enhanced microcalcification lesions, we assessed the morphology pattern of enhancement. Two main patterns of contrast enhancement were evaluated; clumped or stippled and heterogeneous in 21 (68\%) women (pattern 1) and ductal, branching, and heterogeneous in 10 (32\%) women (pattern 2).

As with the level of enhancement we found slow initial peak in $2(6 \%)$ enhanced DCIS, medium peak in $8(27 \%)$ and rapid peak in $20(67 \%)$ of them. In only 1 (3\%) patient with low-grade DCIS we evaluated persistent enhancement pattern. In 20 (67\%) of the DCIS, we assessed the plateau enhancement pattern (Fig. 1, 3) and in $9(30 \%)$ DCIS we found the washout enhancement pattern (Fig. 2) (Table 1).

In one case of $\mathrm{ADH}$ we found the medium initial rise of enhancement and the plateau enhancement pattern (Table 1). Microcalcification lesions with rapid peak and type 2 or 3 of enhancement pattern were considered suspicious of malignancy.

As with the enhancement features of DCIS of various histopathological grades, significant difference was found between the group of low grade and high grade DCIS $(p=0.021)$ and between the group of low grade DCIS and DCIS with microinvasion $(\mathrm{p}=0.006)$ (Table 2).

Altogether in 11 (35\%) patients, we proved the changes (larger size, multifocality) of microcalcification lesions in breast MRI in comparison to MG. In 8 (26\%) patients breast MRI gave the most important information to convert the breast conserving surgery to mastectomy.

\section{DISCUSSION}

Magnetic resonance imaging (MRI) is also used in breast imaging. The evaluation of breast MRI should be done by radiologists who are experienced with other imaging methods used for the breast as well. The assessment of MRI together with other imaging methods mostly mammography, increases the sensitivity and specificity of this method greatly ${ }^{3}$.

Breast MRI has definite indications, and its use should be carefully considered by the radiologist. This examination should not substitute mammography or ultrasound examination even in clear indications for making diagnostic interventions. The only one indication for native breast MRI is evaluating of the integrity of the silicone 

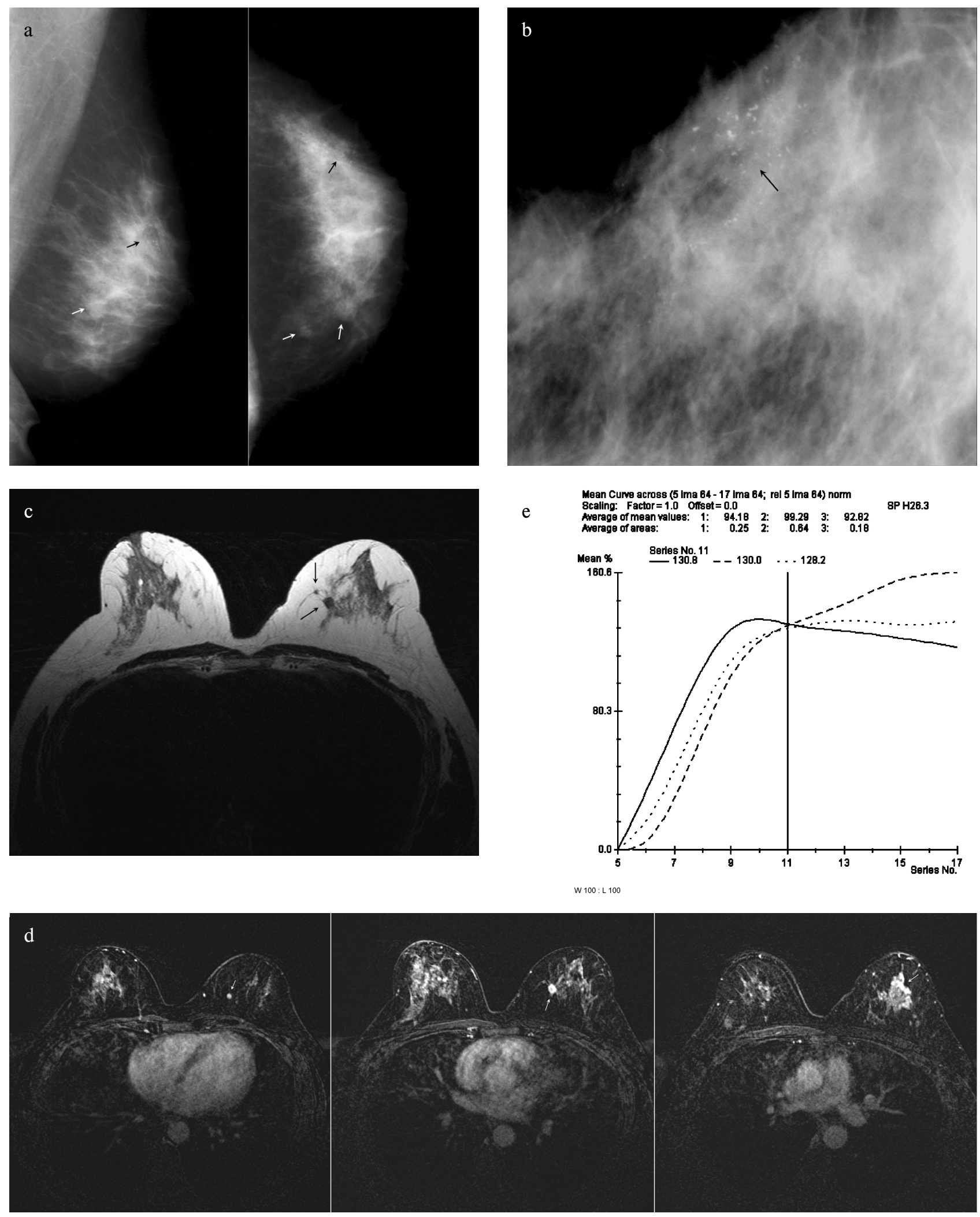

Fig. 1. Woman 43 years of age, screening mammography of the left breast. (a) Microcalcification in the upper outer quadrant (black arrow) and small nodules in the lower inner quadrant (white arrow) of the left breast. (b) Spot compression magnification mammogram demonstrate microcalcifications classified as BI-RADS category 5 (black arrow). (c) Transverse native T2 TSE sequence reveals the small round hypointense lesions in the left breast (black arrows). The area of microcalcification lesion is not visible. (d) Transverse contrast-enhanced T1 FLASH 3D MRI, subtraction image. Small hyperintense round lesions (left and middle images, white arrows) and the clumped and heterogeneous enhancement of microcalcification lesion (right image, white arrow) in the left breast. (e) Time to intensity curve. The rapid initial rise and plateau pattern (type 2) of enhancement (the line curve, the small dotted curve). Final histology was multifocal high - grade DCIS with microinvasion. 

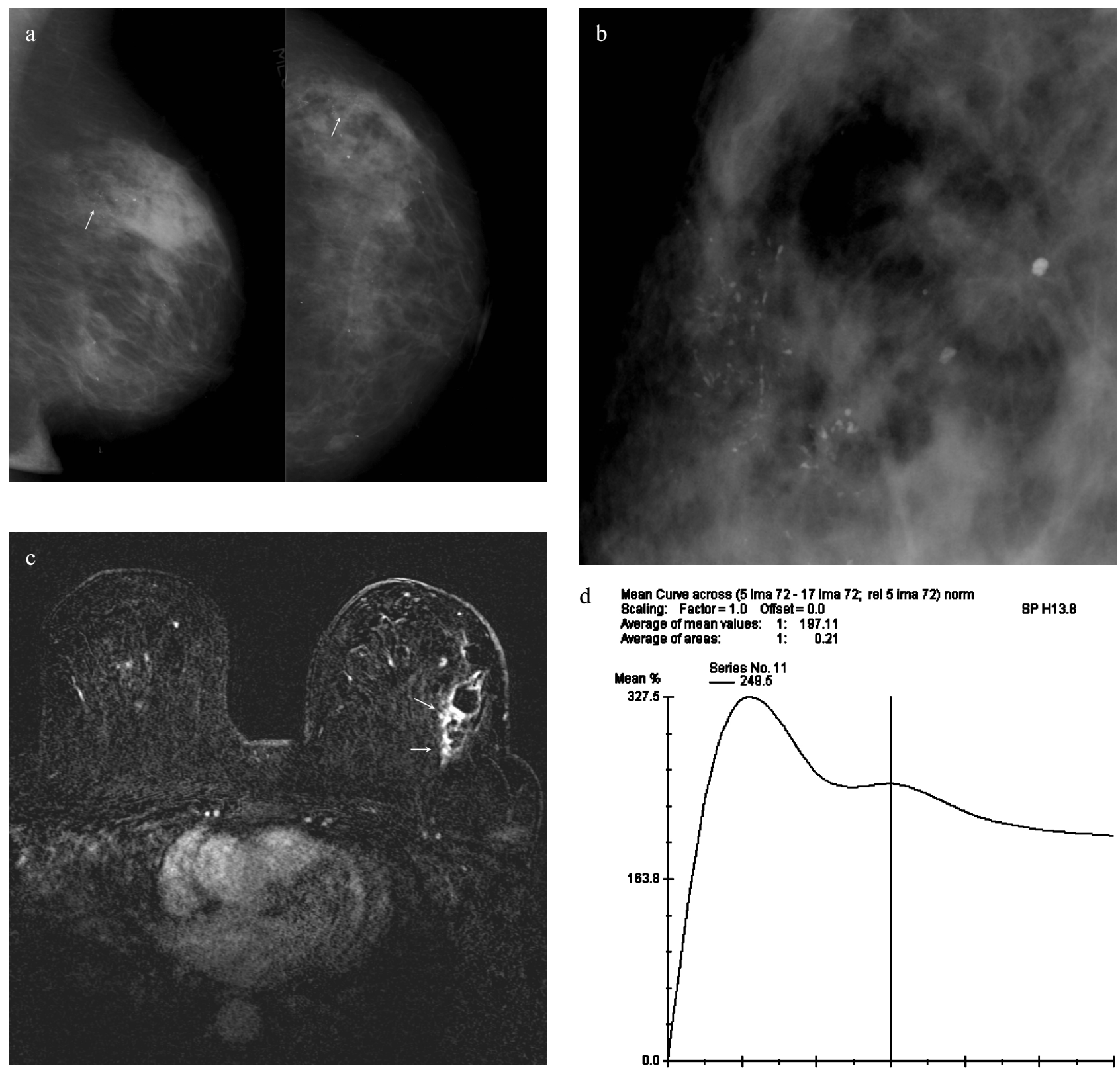

d Mean Curve across (5 Ima 72 - 17 Ima 72; rel 5 Ima 72) norm

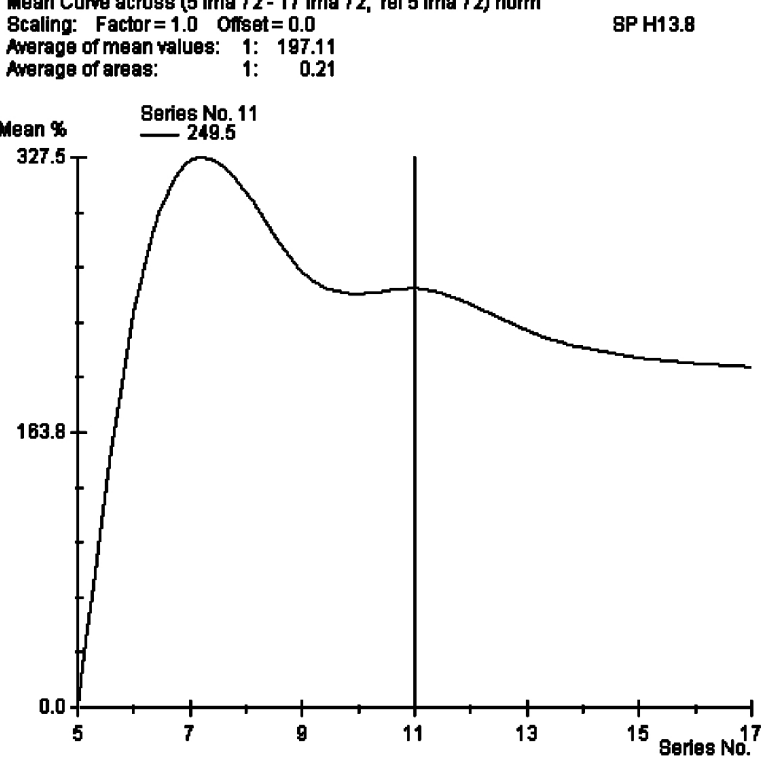

$W 100:$ L 100

Fig. 2. Woman 51 years of age, screening mammography of the left breast. (a) Microcalcification lesion in the upper outer quadrant of the left breast (white arrow). (b) Spot compression magnification mammogram of BI-RADS 5 microcalcification lesion. (c) Transverse contrast-enhanced T1 FLASH 3D MRI, subtraction image. Stippled, clumped and heterogeneous enhancement of microcalcification lesion (white arrows). (d) Breast MRI, time to intensity curve. Rapid initial rise and the washout pattern (type 3) of enhancement. Final histology was intermediate - grade DCIS.

implants. In all other indications breast MRI is always a contrast enhanced dynamic examination with application of contrast media.The main indications for contrast-enhanced dynamic breast MRI are, the suspicion of multifocality or bilateral incidence of carcinoma mainly in dense type of breast or suspicion of presence of carcinoma in breast after augmentation, metastasis in axillary lymph node, negative mammography and negative ultrasound finding. Another indication for breast MRI is, discrimination of residual carcinoma and changes of breast after conservative operation and radiotherapy. In this case it is necessary to examine in intervals of 3-6 months after operation, and 18 months after radiotherapy. Breast MRI is used for screening young high risk dispensary women with dense breast and strong family history of carcinoma and for those who have genes BRCA 1, BRCA 2 or mutation TP 53 (ref. ${ }^{4}$ ). With the help of MRI, it is possible to evaluate the effect of chemotherapy by non adjuvant treatment and not only by the change in size of the lesion but also by the changes in vascularisation of the lesion which demonstrate the changes in kinetic curve assessment. Contraindications for breast MRI are ferromagnetic 

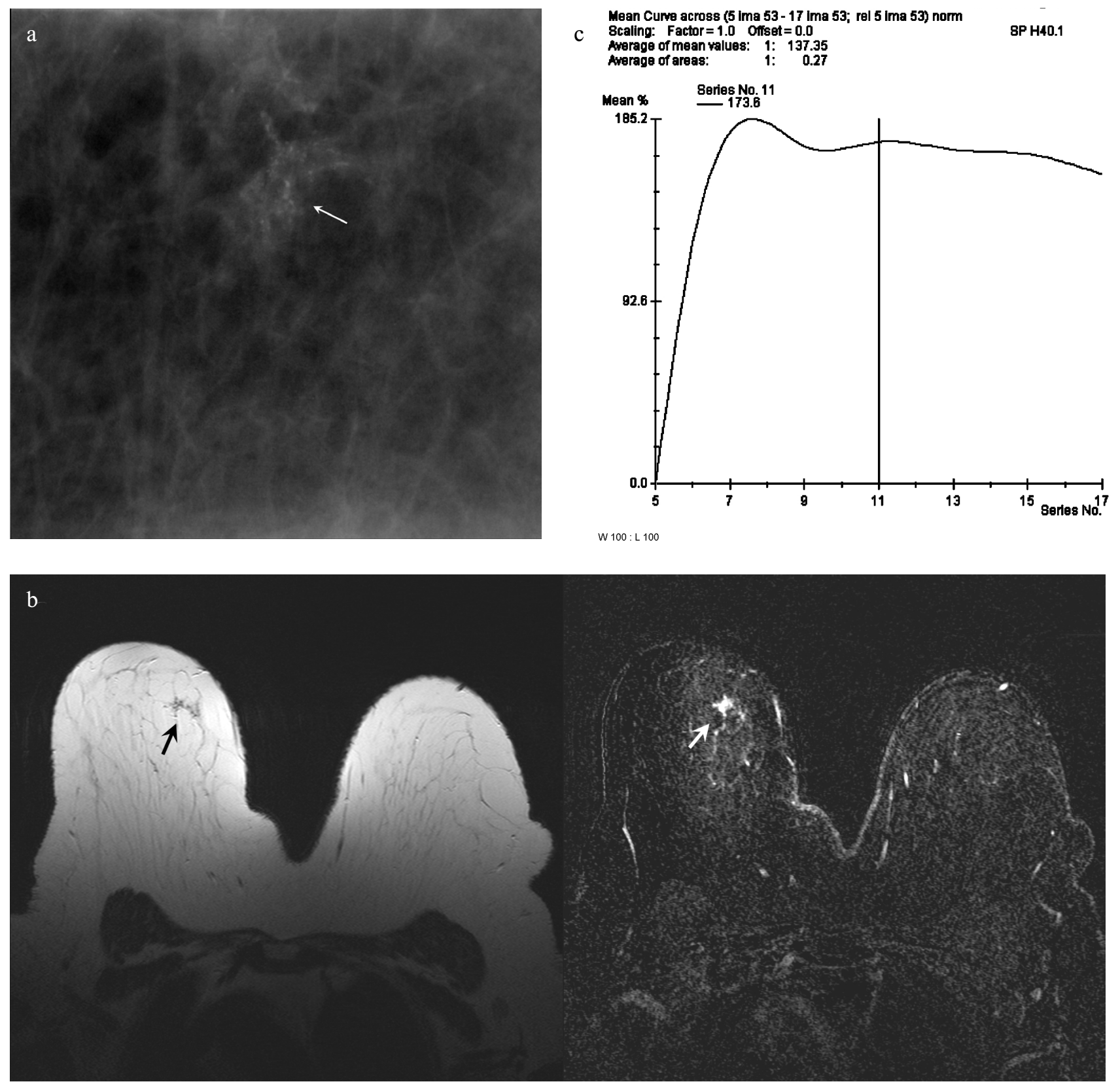

Fig. 3. Woman 38 years of age. Suspicious microcalcifications in the screening mammography of the right breast. (a) Spot compression magnification mammogram of BI-RADS 4 microcalcification in the right breast (white arrow). (b) Transverse native T2 TSE sequence reveals small hypointense irregular area of microcalcification lesion (left image, black arrow). Clumped and heterogeneous enhancement of microcalcification lesion (white arrow, right image) in transverse contrast-enhanced T1 FLASH 3D MRI, subtraction image. (c) Breast MRI, time to intensity curve. The rapid initial rise and the washout pattern of enhancement. Final histology was intermediate - grade DCIS.

material in patients body, claustrophobia, pregnancy and lactating women.

Previously MRI had big changes which were related with the progressing of new examination MR technique. The optimal power of the magnet for examination of the breast is from 1.5 Tesla. It is better to use special double breast coil. In this way examination of both the breasts are done and it is possible to compare them. During the examination, the breasts are fixed in the hole of double breast coil. Dynamic echo sequences with application of contrast media in an amount of $0.1 \mathrm{mmol} / \mathrm{kg}$ are provided in transverse and coronal planes and eventually completed by sagittal plane scanning. Axial plane is used for good imaging of retroareolar and prepectoral areas, in coronal plane there is better visualisation of axillary and parasternal areas. Dynamic contrast study usually consists of native sequence and another 6 (eventually 12) post-contrast sequences. A part of the examination is postprocessing by creating subtraction images and maximum intensity projection (MIP) images. Breast MRI is an im- 
Table 1. Detailed study of the parameters of enhancement according to the histology of microcalcification lesions.

\begin{tabular}{|c|c|c|c|c|c|c|c|c|c|c|}
\hline \multirow{2}{*}{\multicolumn{3}{|c|}{$\begin{array}{l}\text { Final histology } \\
35 \text { patients }\end{array}$}} & \multirow{2}{*}{\multicolumn{2}{|c|}{ Enhancement }} & \multicolumn{3}{|c|}{$\begin{array}{l}\text { MRI enhancement } \\
\text { Initial rise (\%) }\end{array}$} & \multicolumn{3}{|c|}{$\begin{array}{l}\text { MRI enhancement } \\
\text { Type of pattern }(\%)\end{array}$} \\
\hline & & & & & Slow & Medium & Rapid & Type 1 & Type 2 & Type 3 \\
\hline & Benign & 2 & No enhancement & & - & - & - & - & - & - \\
\hline & $\mathrm{ADH}$ & 1 & Enhancement & & - & 1 & - & - & 1 & - \\
\hline \multirow{4}{*}{$\stackrel{\mathscr{\varrho}}{\varrho}$} & \multirow{2}{*}{ Low grade } & \multirow{2}{*}{6} & No enhancement & & - & - & - & - & - & - \\
\hline & & & Enhancement & 4 & 1 & 3 & - & 1 & 3 & - \\
\hline & Intermediate & 9 & Enhancement & & 1 & 3 & 5 & - & 7 & 2 \\
\hline & High grade & 7 & Enhancement & & - & 1 & 6 & - & 4 & 3 \\
\hline & $\begin{array}{c}\text { DCIS } \\
\text { vith microinvasion }\end{array}$ & 10 & Enhancement & & - & 1 & 9 & - & 6 & 4 \\
\hline
\end{tabular}

Table 2. Enhancement parameters of DCIS of different histopathological grades.

\begin{tabular}{|l|c|c|c|c|c|c|c|}
\hline \multirow{2}{*}{$\begin{array}{c}\text { Histological grade } \\
\text { of DCIS }\end{array}$} & \multicolumn{3}{c|}{$\begin{array}{c}\text { MRI enhancement } \\
\text { Initial rise (\%) }\end{array}$} & \multicolumn{3}{c|}{$\begin{array}{c}\text { MRI enhancement } \\
\text { Type of pattern (\%) }\end{array}$} \\
\cline { 2 - 8 } & Slow & Medium & Rapid & Type 1 & Type 2 & Type 3 \\
\hline Low grade DCIS & 4 & 25 & 75 & - & 25 & 75 & - \\
\hline Intermediate grade DCIS & 9 & 11 & 33 & 56 & - & 78 & 22 \\
\hline High grade DCIS & 7 & - & 14 & 86 & - & 57 & 43 \\
\hline $\begin{array}{l}\text { High grade DCIS } \\
\text { with microinvasion }\end{array}$ & 10 & - & 10 & 90 & - & 60 & 40 \\
\hline
\end{tabular}

aging method which differs from other methods used for breast imaging, because it insists not only on the imaging of morphology of lesions, but also on the functional evaluation. Evaluation of enhancement from quantitative and qualitative point of view is infact the evaluation of vascularisation of the lesion. The principle of dynamic contrast enhanced breast MRI is based on the feature of neoangiogenesis of malignant lesion which is sometimes too small to be detected by another imaging method (MG, USG). Enhancement of normal breast during menstrual cycle, dramatically fluctuate by the influence of hormone levels. In premenopausal women, performing breast MRI within the second week of the menstrual cycle may improve the sensitivity and specificity of dynamic contrast - enhanced breast MRI because diffuse enhancement such as unidentified breast objects (UBOś) are reduced.

Breast MR is an imaging method, which has high sensitivity (in invasive carcinoma 95-100\%) and relatively low specificity (40-80\%). The problem with the significance of dynamic contrast enhanced MRI in patients with suspicious microcalcifications of the breast is still being discussed. Of course MRI is unable to image microcalcifications of the breast. Its great importance is in the imaging of a mammographically occult microcalcification lesion of breast. Clustered malignant microcalcifications are the most common presentation of DCIS in mammography. The great benefit of preoperative breast MRI is in imaging the surrounding DCIS with microinvasion and ductal invasive carcinomas that were already diagnosed by mammography. The other benefit of preoperative MRI is assessing multifocality and bilateral incidence of the carcinoma $^{5-7}$.

The sensitivity, specificity and accuracy of breast MR imaging in detecting DCIS with microinvasion were found high in some studies, $71 \%, 85 \%$ and $76 \%$, respectively ${ }^{8}$. Particularly in dense type of breast during MRI, the extent 
of microcalcification lesion can be bigger in comparison with that of MG and it is possible to detect another mammographically and ultrasonographically occult foci of DCIS and observe more accurately the tumour size s, 9-11. $^{\text {. }}$

Microcalcifications as other lesions of the breast are categorized according to BI-RADS. Microcalcifications classified as BI-RADS 4 and 5 are recommended to undergo a biopsy. It is quite difficult to determine the BI-RADS 3 microcalcifications. The short-term mammographic follow-up which is recommended for probable benign lesions is questionable in this case. Recent studies suggest that, histological evaluation of BI-RADS 3 microcalcification is necessary until a more reliable system for the description and classification of these microcalcifications is available. The last study of Japanese authors recommends BI-RADS 3 microcalcifications to be evaluated by MRI prior to SVAB especially in those women who does not want to undergo biopsy ${ }^{22}$. This is due to the high sensitivity of breast MRI for the assessment of malignant and benign microcalcifications ${ }^{12}$.

In literatures, high percentage of agreement regarding the size of malignant lesion detected in breast MRI with definitive histology is mentioned ${ }^{11}$. MRI is not an absolutely reliable method and overestimation and underestimation of the size of the lesion is found ${ }^{13,14}$. It was found that quite a high percentage of re-operations in cases of DCIS was diagnosed only by MG. MRI is understood to be an invaluable adjunct in the evaluation of DCIS or invasive carcinoma especially in those women who consider breast conserving therapy $5,6,9,10$.

Very typical morphological feature for high but also for low DCIS is ductal pattern of enhancement ${ }^{7,15,16}$.Usually it is clumped, and the heterogeneous or homogeneous enhancement sometimes can be of branching appearance and follows the course of the duct ${ }^{8}$. The enhancement can also have a non regular stippled, spotty and micronodular appearance. Segmental and linear character of this enhancement is described and this morphological finding was assessed to have high PPV for DCIS lesions ${ }^{16,17}$. It was assessed that MRI morphologic features of DCIS can reflect differences in biology and pathology of these tumours ${ }^{18}$. The region of interest (ROI) which is necessary to gain relevant quantitative and qualitative evaluation of enhancement must be very small in this ductal pattern of enhancement to obtain the most accurate measurement. The ROI must cover only part of the lesion, and should be placed in the region of strongest enhancement on the first contrast - enhanced image. When the ROI is randomly placed in the mass, the enhancement curve may be variable and yield lower specificity ${ }^{16}$.

However, regarding the detection of ductal carcinoma in situ (DCIS), the sensitivity of MRI varies between $70 \%$ and $100 \%$ according to the latest studies $9,11-13,15,19$. According to Menell's study, breast MRI was significantly more sensitive than mammography in DCIS detection ${ }^{7}$. It was shown that, MRI had relatively high sensitivity for high grade DCIS which often showed rapid initial rise and 2 or 3 of enhancement pattern and thus resembles invasive carcinoma by its characteristics. Low grade DCIS had often quite nontypical form of enhancement pattern and initial rise was usually lower and is of course difficult to differentiate it from benign proliferative processes that often enhance on MRI (ref. ${ }^{20,21}$ ). To Mariano et al. study contrast-enhanced MRI using parametric mapping technique was useful in identifying all intermediate and high-grade DCIS lesions ${ }^{10}$. Regarding the kinetic curve assessment of DCIS, the rapid initial peak of enhancement was found in almost $77 \%$ and washout pattern of enhancement in nearly $54 \%$ of women ${ }^{13}$.

If the ductal pattern of enhancement (during negative MG and USG finding) and kinetic curve assessment suspicious of malignancy are found in MRI, most of the authors recommend biopsy under MRI control ${ }^{3,15}$. Some authors recommend further work-up in case only if the segmental or ductal pattern of enhancement is identified on breast MRI. They recommend either direct MR-guided vaccum-assisted biopsy or short-term follow-up breast MRI within 3 months ${ }^{15}$. Libermann et al. found that the PPV of biopsy for lesions identified in breast MRI significantly increased with increasing size of the lesion and biopsy is rarely necessary for lesions smaller than $5 \mathrm{~mm}$ because of their low likelihood of cancer ${ }^{19}$. If the diagnostic centre for breast is interested in MRI examination seriously, it should be well equipped for doing biopsy and localization under MRI control. It is highly sensitive and indicated in the hands of the experts in breast imaging and upon the correlation with MG and USG.

\section{CONCLUSION}

1. Contrast dynamic - enhanced MRI had sensitivity of $94 \%$, PPV of $100 \%$, NPV of $50 \%$ and accuracy of $100 \%$ for detection of DCIS in our group of BI-RADS 5 microcalcification lesions.

2. Larger size of microcalcification lesion in breast MRI in comparison with MG was proved in $32 \%$ of women. In other $68 \%$, the size of microcalcification lesion in breast MRI and MG was the same.

3. In $19 \%$ of DCIS, we found multifocality of malignant lesion, and bilateral carcinoma was proved in one patient among these women.

4. In $94 \%$ of women with malignancy or $\mathrm{ADH}$, we proved enhancement of microcalcification lesions in breast MRI. Two main patterns of contrast enhancement were evaluated that were typical for DCIS microcalcificaton lesions. In DCIS rapid initial rise of enhancement (in $67 \%$ ), and the plateau (in $67 \%$ ) or washout (in $30 \%$ ) enhancement patterns were found most frequently.

5. As with MRI enhancement features, noted differences between the low grade and high grade DCIS $(p=0.021)$ and low grade and high grade DCIS with microinvasion, ( $\mathrm{p}=0.006)$ were found.

6. In $26 \%$ of women, the result of breast MRI was the most important to convert the breast conserving operation to mastectomy. 


\section{ACKNOWLEDGEMENT}

This study is supported by grant IGA Ministry of Health Czech Republic, No. NR-7881-3/2004.

\section{REFERENCES}

1. Fischer U. MR - guided interventions. In: Fischer U. Practical MR Mammography. Stuttgart-New York: Georg Thieme Verlag, 2004: 181-191.

2. Daneš J. Mamografická typologie. In: Daneš J. et al. Základy mamografie. X-Egem, 2002: 96-101.

3. Schnall MD, Blume J, Bluemke DA, et al. Diagnostic architectural and dynamic features at breast MR imaging: multicenter study. Radiology 2006; 238: 42-53.

4. Leach MO, Boggis CR, Dixon AK, et al; MARIBS study group. Screening with magnetic resonance imaging and mammography of a UK population at high familial risk of breast cancer; a prospective multicenter cohort study (MARIBS). Lancet 2005; 365: 1747-9.

5. Chung A, Saouaf R, Scharre K, Phillips E. The impact of MRI on the treatment of DCIS. Am Surg 2005; 71: 705-10.

6. Hwang ES, Kinkel K, Esserman LJ, Lu Y, Weidner N, Hylton NM. Magnetic resonance imaging in patiens diagnosed with ductal carcinoma in situ: value in the diagnosis of residua disease, occult invasion, and multicentricity. Ann Surg Oncol 2003; 10: 381-8.

7. Menell JH, Morris EA, Dershaw DD, Abramson AF, Brogi E, Liberman L. Determination of the presence and extent of pure ductal carcinoma in situ by mammography and magnetic resonance imaging. Breast J. 2005; 11: 382-90.

8. Ikeda $\mathrm{O}$, Nishimura R, Miyayama $\mathrm{H}$, et al. Magnetic resonance evaluation of the presence of an extensive intraductal component in breast cancer. Acta Radiol 2004; 45: 721-5.

9. Shiraishi A, Kurosaki Y, Maehara T, Suzuki M, Kurosumi M Extension of ductal carcinoma in situ: histopathological association with MR imaging and mammography. Magn Reson Med Sci 2003; 2 : 159-63.

10. Smith M, Allison K, Shaw de Paredes E. Nonmammographic evaluation of the extent of breast carcinoma. Semin Ultrasound CT MR 2006; 27: 308-19.
11. Zuiani C, Francescutti GE, Konsero V, Zunnui I, Bazzocchi M. Ductal carcinoma in situ: is there a role for MRI? J Exp Clin Cancer Res 2002; 21: 89-95.

12. Kneeshaw PJ, Lowry M, Manton D, Hubbard A, Drew PJ, Turnbull LW. Differentiation of benign from malignant breast disease associated with screening detected microcalcifications using dynamic contrast enhanced magnetic resonance imaging. Breast 2005; 15: 29-38.

13. Bazzocchi M, Zuiani C, Panizza P, et al. Contrast-enhanced breast MRI in patients with suspicious microcalcifications on mammography: results of a multicenter trial. AJR Am J Roentgenol 2006; 186: $1723-32$.

14. Schouten van der Velden AP, Boetes $C$, Bult $P$, Wobbes $T$. The value of magnetic resonance imaging in diagnosis and size assessment of in situ and small invasive breast carcinoma. Am J Surg 2006; 192: $172-8$.

15. Van Goethem M, Schelfout K, Kersschot E, et al. Comparison of MRI features od different grades of DCIS and invasive carcinoma of the breast. JBR-BTR 2005; 88: 225-32.

16. Katarzyna J, Ouwerkerk R, Jacobs M, et al. Patterns of enhancement on breast MR images: Interpretation and Imaging Pitfalls. RadioGraphics 2006; 26: 1719-1734.

17. Morakkabati-Spitz N, Leutner C, Schild H, Traeber F, Kuhl C. Diagnostic uselfulness of segmental and linear enhancement in dynamic breast MRI. Eur Radiol 2005; 15: 2010-7.

18. Esserman LJ, Kumar AS, Herrera AF, et al. Magnetic resonance imaging captures the biology of ductal carcinoma in situ. J Clin Oncol 2006; 24: 4603-10.

19. Mariano MN, van den Bosch MA, Daniel BL, et al. Contrast- enhanced MRI of ductal carcinoma in situ: characteristic of a new intensity - modulated parametric mapping technique correlated with histopathologic findings. J Magn Reson Imaging 2005; 22: 520-6.

20. Kumar AS, Chen DF, Au A, et al. Biologic significance of falsepositive magnetic resonance imaging enhancement in the setting of ductal carcinoma in situ. Am J Surg 2006; 192: 520-4.

21. Liberman L, Mason G, Morris EA, Dershaw DD. Does size matter? Positive predictive value of MRI detected breast lesions as a function of lesion size. AJR Am J Roentgenol 2006; 186: 426-30.

22. Uematsu T, Yuen S, Kasami M, Uchida Y. Dynamic contrast-enhanced MR imaging in screening detected microcalcification lesions of the breast: is there any value? Breast Cancer Res Treat 2007; 103:269-81. 